\title{
EARLY DECREASE OF TYPE 1 CANNABINOID RECEPTOR BINDING AND PHOSPHODIESTERASE 10A ACTIVITY IN VIVO IN R6/2 HUNTINGTON MICE
}

Maarten Ooms ${ }^{1,2}$, Roma Rietjens ${ }^{2,3}$, Janaki Raman Rangarajan ${ }^{4}$, Kathleen Vunckx ${ }^{3}$, Sara

Valdeolivas ${ }^{5}$, Frederik Maes ${ }^{4}$, Uwe Himmelreich ${ }^{2,6}$, Javier Fernandez-Ruiz ${ }^{5}$, Guy Bormans ${ }^{1,2}$, Koen van Laere ${ }^{2,3}$, Cindy Casteels ${ }^{2,3}$

${ }^{1} \mathrm{KU}$ Leuven Laboratory of Radiopharmacy, Herestraat 49, 3000 Leuven, Belgium

${ }^{2}$ KU Leuven Molecular Small Animal Imaging Centre (MoSAIC), Herestraat 49, 3000 Leuven, Belgium

${ }^{3} \mathrm{KU}$ Leuven and University Hospital Leuven Division of Nuclear Medicine and Molecular Imaging, Herestraat 49 bus 505, 3000 Leuven, Belgium

${ }^{4} \mathrm{KU}$ Leuven Medical Image Computing (ESAT/PSI) Department of Electrical Engineering \& Medical Imaging Research Center, Herestraat 49, 3000 Leuven, Belgium

${ }^{5}$ Universidad Complutense de Madrid Departamento de Bioquímica y Biología Molecular, Facultad de Medicina, Madrid,Spain

${ }^{6}$ KU Leuven Biomedical NMR Unit, Herestraat 49, 3000 Leuven, Belgium

\section{Introduction}

Several lines of evidence imply early alterations in endocannabinoid and phosphodiesterase 10A (PDE10A) signaling in Huntington's disease (HD). Using $\left[{ }^{18} \mathrm{~F}\right] \mathrm{MK}-9470$ and $\left[1^{8} \mathrm{~F}\right] J N J 42259152$ small-animal PET, we investigated for the first time cerebral changes in type 1 cannabinoid (CB1) receptor binding and PDE10A levels in vivo in pre-, early- and late symptomatic $\mathrm{HD}(\mathrm{R} 6 / 2)$ mice, in relation to brain morphology (MRI) and motor function.

\section{Methods}

Ten R6/2 and 16 wild-type (WT) mice were investigated at 3 different time points between the age of 4 and 13 weeks. Parametric CB1 receptor and PDE10A images were anatomically standardized to Paxinos space and analyzed voxel-wise. Volumetric microMRI imaging was performed to assess HD pathology.

\section{Results}

In R6/2 mice, CB1 receptor binding was decreased in comparison to WT in the bilateral caudateputamen, globus pallidus and thalamic nucleus at week $5\left(-8.1 \%\right.$, $\left.p_{\text {height }}=1.7 .10^{-5}\right)$. Longitudinal follow-up showed further progressive decline compared to controls in a cluster comprising the bilateral hippocampus, caudate-putamen, globus pallidus, superior colliculus, thalamic nucleus and cerebellum (late vs. presymptomatic age: $-13.7 \pm 3.1 \%$ for $R 6 / 2$ and $+1.5 \pm 4.0 \%$ for WT; $p_{\text {height }}=1.9 .10^{-5}$; Fig. 1 ). In R6/2 mice, PDE10A binding potential also decreased over time, to reach significance at early and late symptomatic HD (late vs. presymptomatic age: $-79.1 \pm 1.9 \%$ for $R 6 / 2$ and $+2.1 \pm 2.7 \%$ for $\mathrm{WT}$; $p_{\text {height }}=1.5 .10^{-4}$; Fig. 2 ). The observed changes in CB1 receptor and PDE10A binding were correlated to anomalies exhibited by $R 6 / 2$ animals in motor function, while no correlation was found with MRI-based striatal volume.

\section{Conclusions}

Our findings point to early regional dysfunctions in endocannabinoid and PDE10A signaling, involving the caudate-putamen and lateral globus pallidus, that may play a detrimental role in the progression of the disease in R6/2 animals. PET quantification of in vivo CB1 and/or PDE10A binding may thus be useful early biomarkers for HD. Our results also provide evidence of subtle motor deficits at earlier stages than previously described.

\section{Acknowledgement / References}

Financial support of the Fund for Scientific Research, Flanders, Belgium (FWO/G.0972.13), and the KU Leuven In Vivo Molecular Imaging (IMIR) Consortium (KUL PF/10/017). Cindy Casteels is supported by a post-doctoral mandate of the Research Foundation Flanders. 


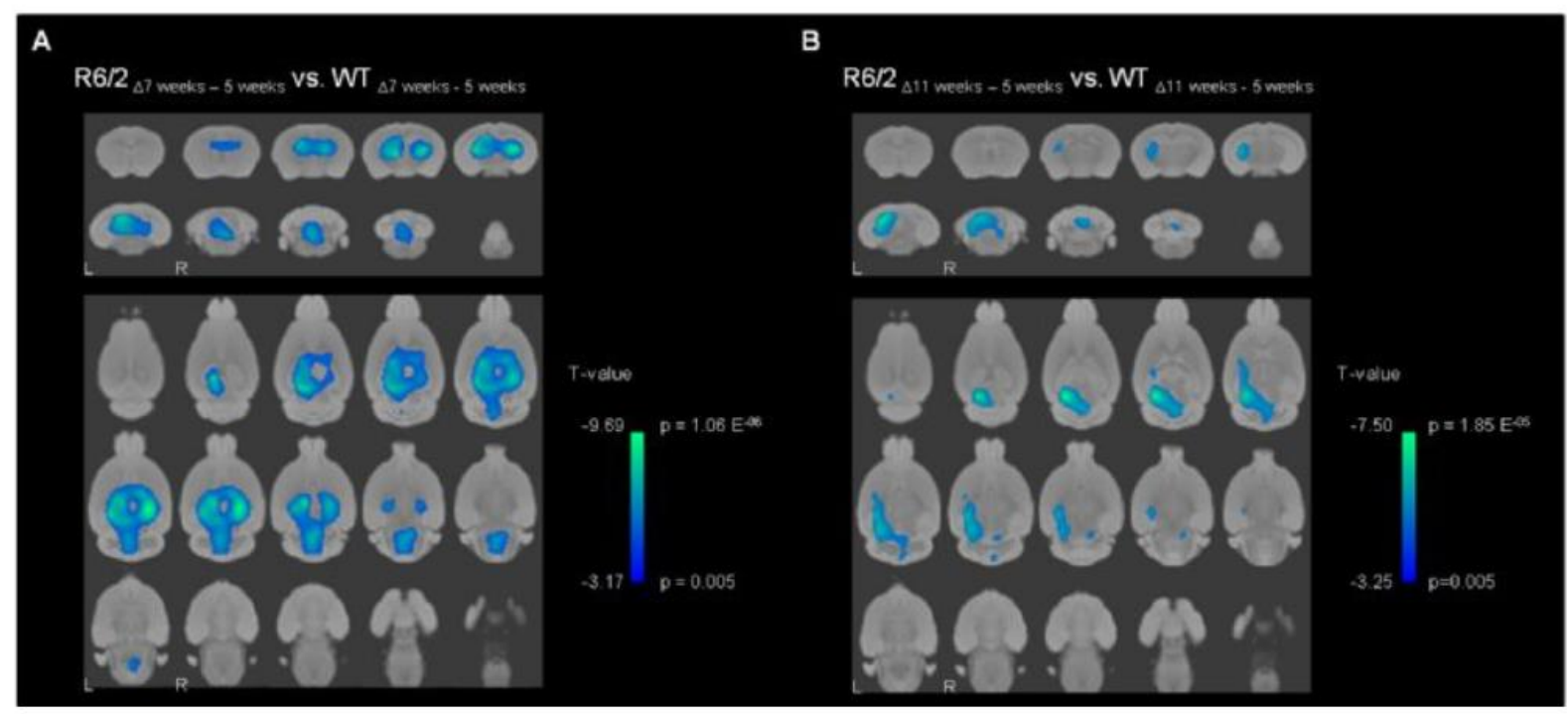

Fig1:CB1 Receptor in R6/2: (A-B) Coronal and axial brain sections showing decreased [18F]MK-9470 binding in R6/ 2 mice over time, as compared to WT littermates. Significance is shown with a T statistic color scale. Images are in neurological convention.

Image 1 of 2

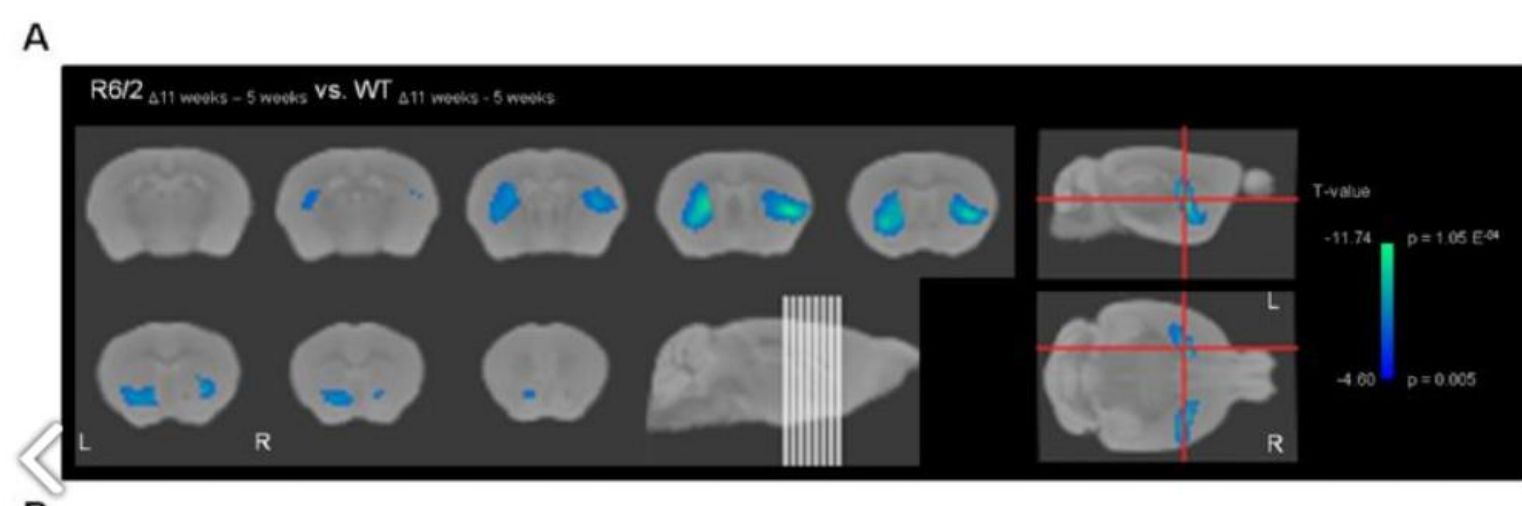

B

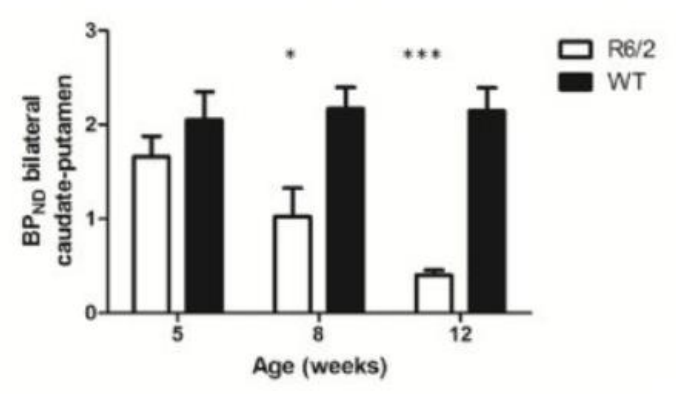

Fig2: PDE10A in R6/2: (A) Coronal brain sections showing decreased PDE10A binding potential in R6/2 mice over time, as compared to WT littermates. (B) Histograms of BP values of the bilateral caudate-

putamen in R6/2 and WT animals over time. 2 -way ANOVA; * $p<0.05 ; * * * p<0.001$

Image 2 of 2 